\title{
Reseña del Libro
}

http://dx.doi.org/10.22402/j.rdipycs.unam.1.2.2015.26.291-295

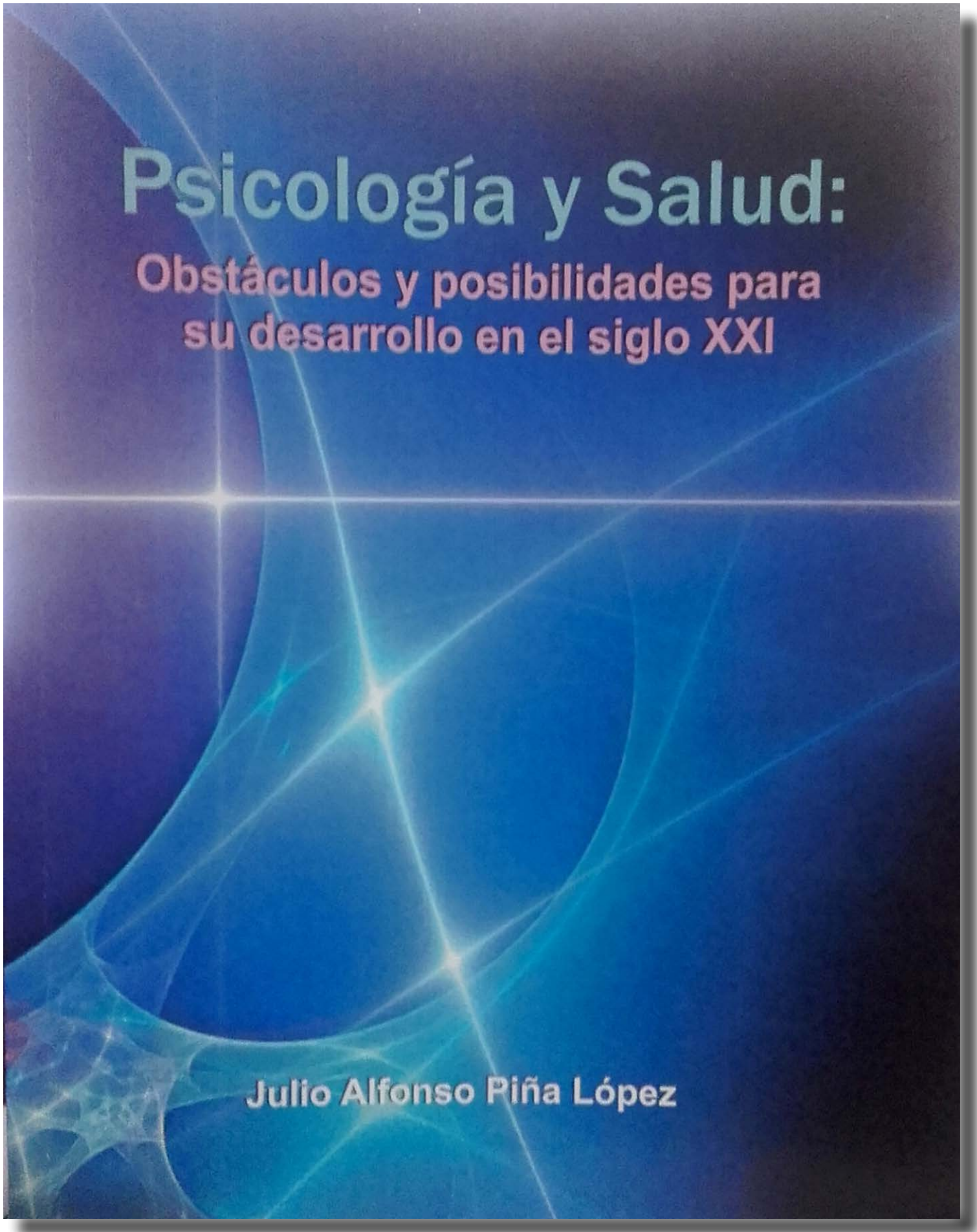




\section{Julio Alfonso Piña López}

Psicología y Salud 


\section{Reseña del libro por}

\section{Sandra Ivonne Muñoz Maldonado}

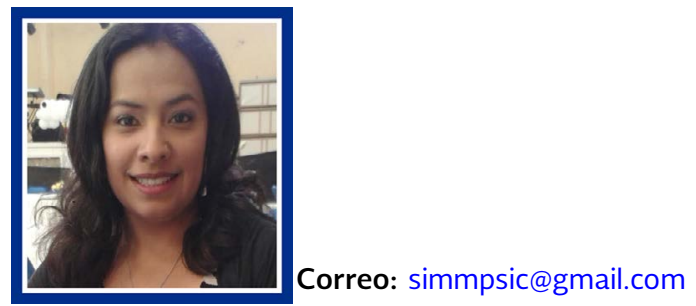

Doctora en Psicología en el área de la Salud por la UNAM, 2016. Licenciada en Psicología por la Facultad de Psicología de la UNAM; miembro del Grupo de Investigación en Procesos Psicológicos y Sociales en la UNAM FES Iztacala.

Ver más... 
E n un país como el nuestro, donde en el sistema de salud ha predominado una visión patologista, que se centra en la cura de enfermedades y no en la prevención de las mismas, es difícil que un psicólogo se inserte y además se reconozca la contribución que puede hacer para la promoción de la salud y la prevención de las enfermedades. Aún cuando la Ley General de Salud de México, publicada en 2007, determina que la investigación en salud debe identificar los procesos tanto biológicos como psicológicos relacionados con la salud, no se establece el rol del psicólogo en la investigación de los mismos. Los psicólogos que se han insertado en el campo tienen dentro de sus actividades las referentes a la evaluación y diagnóstico de trastornos psicológicos, funciones propias de un psicólogo clínico, de manera que el propio sistema de salud no contempla que el psicólogo es capaz de aportar en este ámbito para explicar por qué la persona enferma, predecir qué conductas se pueden modificar para prevenir que enferme, y llevar a cabo intervenciones preventivas y acciones de promoción de la salud.

Es necesario resaltar que el contexto de salud en México está definido por un aumento en enfermedades crónicas no transmisibles, asimismo que los costos que estas enfermedades causan a las instituciones de salud pública están mermando los recursos económicos e impactan en el desarrollo del país, dando como resultado mayor ausentismo laboral y pobre desarrollo personal debido a complicaciones cardiovasculares, hipertensión, diabetes, etc. De seguir con este panorama llegará un momento en que el sistema de salud no pueda atender a todas aquellas personas con una enfermedad crónica, degenerativa; por ello es importante redirigir los esfuerzos no sólo al tratamiento o control de las enfermedades, sino más bien enfocarse a la prevención de dichas enfermedades y promover conductas que hagan permanente el estado de salud del individuo, por lo que el libro que se presenta a continuación aborda y expone la relación de la psicología y la salud, y los factores a considerar para su consolidación en México.

El libro Psicología y salud: obstáculos y posibilidades para su desarrollo en el siglo XXI, es una obra realizada por el M. en C. Alfonso Piña; ésta fue publicada en el año 2015 por la Universidad de Sonora. El objetivo principal del libro es hacer una reflexión y una crítica sustentada en evidencias sobre aspectos relacionados con la psicología y la salud, que de forma general el autor delinea en tres puntos: el primero se refiere a la diferenciación de la psicología clínica y la psicología en el campo de la salud; el segundo alude al origen y características de los modelos teóricos utilizados en la psicología de la salud, y el tercero se enfoca en las competencias profesionales que debe desempeñar un psicólogo en esta área, centrándose en la prevención de la enfermedad y la promoción de la salud.

Esta obra está estructurada en cuatro capítulos que retoman los aspectos mencionados; en el primer capítulo se hace un recorrido por los antecedentes que se han marcado de forma tradicional como los principales ejes o hechos para el surgimiento de la psicología de la salud, se examinan la superposición de la psicología clínica y de la salud en términos de sus funciones, y se propone la diferenciación de ambas demandando tener claridad respecto a lo que la psicología aporta con relación a la salud, y no caer en el eclecticismo teórico que permea las definiciones actuales.

En el segundo capítulo se definen diversos conceptos como "modelo teórico", "paradigma" y "teoría" para dar paso a la definición de "psicológico" desde la visión del autor, y cómo es que lo psicológico se relaciona con los fenómenos de salud y enfermedad; apela a una propuesta teórica articulada en lo conceptual que posibilite la predicción de las conductas que favorecen la salud o que previenen que las personas adquieran enfermedades. En la segunda parte del capítulo se examinan modelos teóricos que se emplean en el campo de la salud, como el de creencias en salud (Rosentock,1974), la acción razonada (Azjen y Fishbein, 1980), biopsicosocial (Engel, 1977), integrado de cambio conductual (Fishbein, 2000); cada uno es analizado, desde la definición de sus variables, su congruencia conceptual y en la evidencia empírica que aporta a través de diversos estudios empíricos.

En el tercer capítulo se insiste en la importancia de lo psicológico en relación con la salud; este último concepto lo revisa y analiza desde diversos puntos de vista, para finalmente aportar una definición propia de la salud basada en la psicología interconductual, lo que permite abordar el modelo de salud biológica (Ribes, 1990), que se revisa de forma crítica y reflexiva desde sus supuestos teóricos, aplicaciones y limitaciones. Una vez establecido el modelo, se delimitan las funciones del psicólogo en la promoción, prevención y rehabilitación. 
El capítulo final retoma aspectos revisados en los tres anteriores para identificar cuáles serían las competencias que debemos formar en los nuevos profesionales en psicología; la propuesta contempla competencias teóricas y metodológicas que se enfocan a planear, evaluar e investigar, para promover, prevenir y rehabilitar en el campo de la salud. Esta empresa —afirma el autor- es un compromiso que se debe cumplir en aras de poder avanzar en la consolidación de este campo de actuación.

A manera de conclusión, este volumen se dirige a población que ya tenga conocimientos acerca del área; no es recomendable para lectores que empiezan a conocer el campo, debido a que es necesario conocer los antecedentes de la psicología de la salud, conocer y manejar los modelos teóricos que relacionan la psicología y la salud; asimismo, debe tener bagaje empírico y teórico que le permita seguir al autor en las disertaciones, reflexiones y propuestas que plantea acerca de los modelos teóricos revisados, para al final Ilevar a cabo una reflexión crítica de lo expuesto en los diferentes niveles de conocimiento, ya sea teórico, metodológico y prácti- co, de forma que el público estaría más bien en los profesionales que ya están insertos en el campo de acción o a los profesores que se encuentran formando profesionales en esta área, para hacer una reflexión de lo que el autor propone y clarificar las metas que se deben alcanzar en la formación de competencias en los jóvenes que están ingresando a las instituciones de educación superior con un programa de psicología que tiene una vertiente en salud.

\section{Referencias}

Azjen, I., \& Fishbein, M. (1980). Understanding attitudes and predicting social behavior. New York, Prentice Hall.

Fishbein, M. (2000). The role of theory in HIV prevention. AIDS Care, 12, 273-278.

Ribes, E. (1990). Psicología y salud: un análisis conceptual. Barcelona, Martínez Roca.

Rosenstock, I. (1974). Historical Origins of the Health Belief Model. Health Education Monographs, 2(4), 328-335.

Ir a Google Books para revisar la tabla de contenido del libro 
La edición en formato electrónico fue realizada en la Editorial LEED del Laboratorio de Evaluación y Educación Digital

Diciembre, 2015

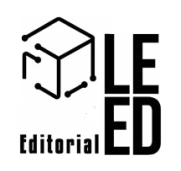




\section{(c) (i) (5) \\ BY NC}

Copyright: @ 2015 Revista Digital Internacional de Psicología y Ciencia Social

Esta es una revista de acceso abierto distribuida bajo los términos de la licencia

, por lo que su contenido gráfico y escrito se puede compartir, copiar y redistribuir total o parcialmente sin necesidad de permiso expreso de sus creadores con la única condición de que no se puede usar con fines directamente comerciales y los términos legales de cualquier trabajo derivado deben ser los mismos que se expresan en la presente declaración. La única condición es que se cite la fuente con referencia a la y a sus creadores.

2015, es una una publicación semestral editada por la Universidad Nacional Autónoma de México, tercer piso del edificio B de la of. admin. ext. de la Zona Cultural, Ciudad Universitaria, Coyoacán, C.P. 04510, Distrito Federal, Tel. (55) 5623-1333, Ext. \#39707,

.Editor Responsable: Arturo Silva Rodríguez. Reserva de Derechos al Uso Exclusivo No. 04-2016-012514394300-203, ISSN: (en trámite), ambos otorgados por el Instituto Nacional del Derecho de Autor. Responsable de la última actualización de este número, Laboratorio de Evaluación y Educación Digital de la Universidad Nacional Autónoma de México (UNAM), Facultad de Estudios Superiores Iztacala, Lic. José Manuel Sánchez Sordo, Av. de los Barrios \#1, Los Reyes Iztacala, Tlalnepantla Edo. de México. C.P. 54090, fecha de última modificación, 25 de enero de 2016. La difusión y soporte digital a cargo del Laboratorio de Evaluación y Educación Digital de la Universidad Nacional Autónoma de México (UNAM), Facultad de Estudios Superiores Iztacala. Las opiniones expresadas por los autores no necesariamente reflejan la postura del editor de la publicación. 